\title{
Distributed Optimal Control of Transient Stability for a Power Information Physical System
}

\author{
Shiming Chen and Kaiqiang $\mathrm{Li}$ (i) \\ East China Jiaotong University, Nanchang 330013, China \\ Correspondence should be addressed to Kaiqiang Li; 601613012@qq.com
}

Received 26 March 2020; Accepted 9 May 2020; Published 26 May 2020

Guest Editor: Michael Z. Q. Chen

Copyright (c) 2020 Shiming Chen and Kaiqiang Li. This is an open access article distributed under the Creative Commons Attribution License, which permits unrestricted use, distribution, and reproduction in any medium, provided the original work is properly cited.

\begin{abstract}
The development of power energy structures and information communication technology has promoted the renewal of smart grid information-physical structures. At the same time, the changes in the smart grid energy structure and the vulnerability of the information network threaten the stability of the power system and uses multiagent control theory to improve the transient stability of the power grid which has strong practicability. In this paper, an optimized distributed control scheme is proposed for application to the smart grid model so that the grid system can flexibly adapt to the external operating conditions and recover to stable operating conditions after being disturbed. In this paper, an intelligent power grid information-physical network simulation system is established. According to the information exchange within the multiagent system, groups of coherent generators in the disturbed power grid in different regions are identified and controlled. Distributed control is applied to maintain the exponential frequency synchronization and phase angle aggregation of the synchronous generators to achieve transient stability. Finally, the effectiveness and rapidity of the proposed distributed optimal control scheme are verified by simulation analysis of the IEEE 39 node model.
\end{abstract}

\section{Introduction}

With the reorganization and renewal of the energy structure in recent years, the standards of smart grid systems in terms of their transient stability have been raised. A smart grid itself is based on the ultra-high-voltage UHV backbone transmission network and deeply integrates more stable information network technology to establish a strong power system network, realizing close connection and integration of the power system and information system [1]. The development of CPSs promotes the deep integration of physical power systems and power information systems. It provides a new way to realize the goal of power grid intelligence [2]. In the smart grid model, the information network dispatching centre node collects and processes the information of each physical power station and sends corresponding control instructions. Each physical power station supplies power to the information node to ensure its normal operation. Through this connection, the mutual dependent relationship between the physical network and the information network is formed (Figure 1) [3]. With the intelligent development of smart grids, there is a dynamic interaction among the five "generation, transmission, supply, distribution, and use" terminals of smart grids with the two-way flow of energy and information [4], new energy, and other distributed energy grid connections and huge data processing between the information network and the physical network. Additionally, the basic characteristics of smart grid informatization, automation, and interaction are the operational belt of the great smart grid challenge $[5,6]$.

Information systems and information networks play an important role in enhancing the safe operation of smart grids. In actual operation, the dependence of the physical system on the information system will significantly affect the stable operation of the physical system in the case of information system failure. In actual operation, the cost of communication and information processing should also be considered. The use of redundant information and weakly 

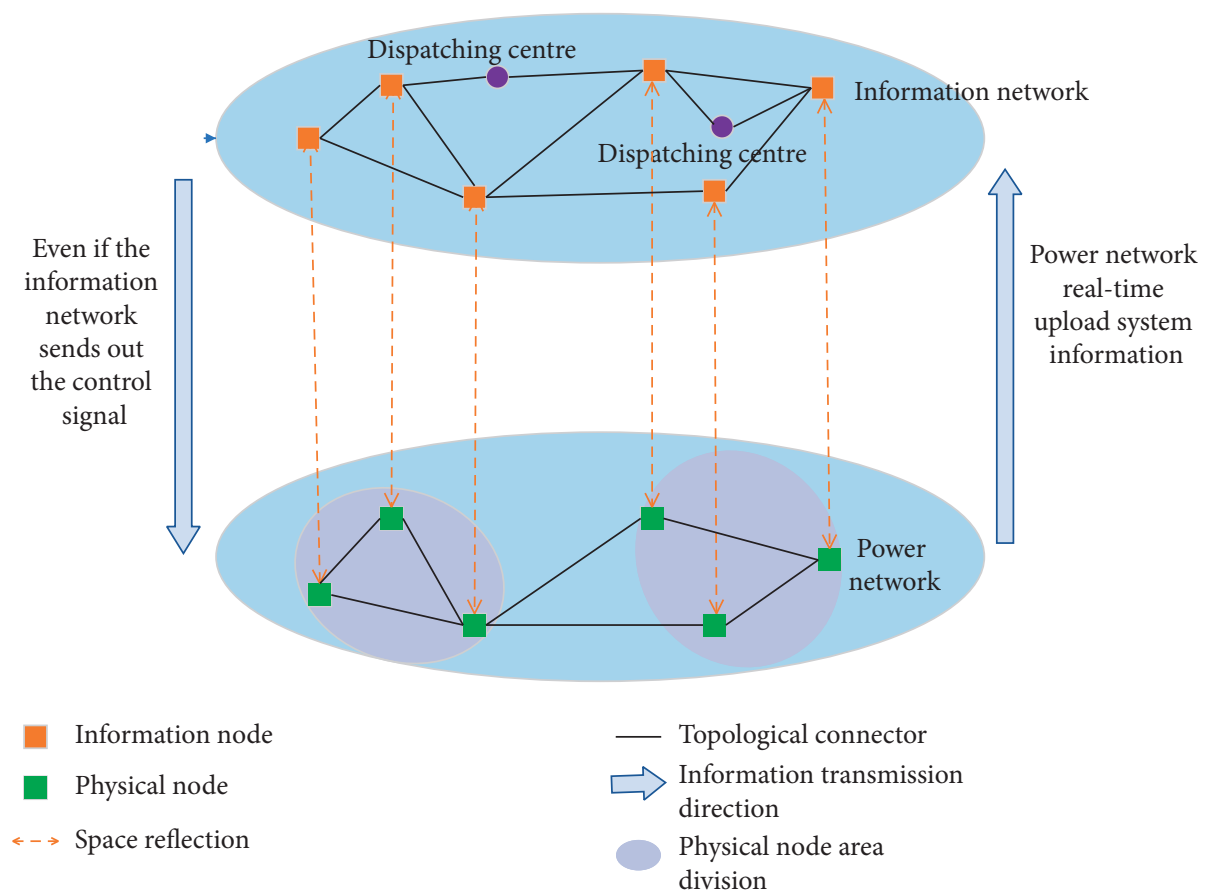

Figure 1: Model of a cyber-physical smart grid.

correlated information in computing will cause a too high computational burden, resulting in poor performance of the system state. The stability and flexibility of smart grids can be effectively improved by determining an appropriate dependency on the information network and information flow. For phasor measurement units (PMUs), which are synchronous phasor measurement devices, a reasonable number of them at suitable locations can synchronously collect the corresponding parameters of the power system at the installation site and achieve the overall observability, economic and reliability goals of the system online state calculation, and system state prediction [7-9]. In this paper, PMUs are introduced into the optimal configuration of the power system network structure to measure the voltage vectors of some nodes, which can improve the dependence on information, providing strong initial conditions and a discrimination basis for system stability analysis, protection, and reconstruction. This is of great significance for system online state calculation and system state prediction.

The traditional power grid control strategy mostly adopts a centralized scheduling method, and the dispatching centre needs to communicate with all generators and load nodes through the information network, with a high degree of communication dependence [10]. The "plug and play" technology required by the grid connection and independent operation of new energy and power components make the topological structure of the information network and physical network of the smart grid changeable [11]. The centralized control strategy has difficulty avoiding the high cost of communication facility construction. Distributed control technology can meet the flexibility requirements and provide high-efficiency control constraints [12]. In [13], a smart grid scheduling strategy considering flexible load is designed, which have better convergence and application prospect compared with the centralized scheduling strategy.

In $[14,15]$, distributed control is used to reduce the cost of the communication network, and power distribution and frequency stability are achieved. Energy storage technology provides a new technology choice for the safe and economic operation of power grids, stabilization of power fluctuations, and distributed control [16-19]. In [16-18], the influence of flywheel energy storage on the power balance and stability of the power grid is studied. In [19], a fast control method for a battery energy storage system is adopted to improve the transient stability of the power grid. In [20], a distributed control method of energy storage system based on real-time wind power output regulation of energy sharing is proposed. In [21], a distributed intelligent power grid controller based on the Robust Consistency Algorithm of the second-order multiagent system is proposed. The controller is composed of a physical topology model of the network. The delay characteristics and anti-interference ability of the controller are verified by the IEEE39 node system.

In [22], the problem of cluster behavior and target consistent tracking of heterogeneous multi-inertial bodies with limited communication distance is studied. A distributed control protocol is designed to enable agents to achieve stable group behavior. In [23], a distributed observer is designed to estimate the relatively complete state of a general linear multiagent system that does not directly enter the real-time state, and it is used to track the consistency protocol, so as to achieve the overall consistency. In [24], a distributed multicluster method based on partial information exchange is designed by studying the multiobjective consistency pursuit of the multiagent system, and sufficient conditions are given to realize the dynamic goal consistency 
pursuit, so that the agent can adaptively select the target for tracking. In [25], we study the problem of consistency tracking of heterogeneous interdependent group systems with fixed communication topology and propose a distributed consistency tracking control protocol, which uses local information to achieve the consistency tracking of heterogeneous interdependent group systems. In [26], a new scale consistency protocol is proposed to solve the problem of time-varying delay in nonlinear dynamics and communication networks. In this paper, based on the rules of speed consistency and group concentration of a multiagent system, the phase angle (rotor angle) aggregation and frequency consistency of power grid generators are realized by using the local and associated node information to inject power into or absorb power from generator nodes through an external energy storage device (such as a flywheel) to ensure synchronous operation of the generators and realize transient stability of the power system.

In the context of transient stability, this paper integrates information and physical nodes into an agent, adopts the distributed control strategy of a "leading assisting" agent, and utilizes the information of local and other regional physical nodes and the coupling relationship of the physical network. It also realizes global information flow and stable operation of the system through the interaction of the information and physical networks and external energy storage. In this paper, the IEEE 39 node grid model is used as an example to demonstrate the effectiveness of the application of optimized distributed control in the smart grid model to maintain the stability of the system [27].

\section{Transient Stability Control of a Smart Grid}

To ensure that the power grid can quickly respond after being disturbed by information and a physical disturbance and reduce the implementation time of transient stability zone control, necessary information network reliability optimization measures are taken to reduce information redundancy and unnecessary calculation and achieve the purpose of real-time control.

2.1. Reliability Optimization of a Smart Grid Information Network. Considering the reliability of the data processing of the information network under the influence of a single line fault in the physical network, the goal of a fully observable power network can be achieved by ensuring the optimal configuration of PMUs (synchronous vector measurement devices) with different channel capacities in the information network. Based on the configuration rule [28] of the minimum number of PMUs in the information network, the PMU optimization method to avoid scheme repetition is adopted, and the model is defined as follows:

$$
\left\{\begin{array}{l}
\min \left(x=\sum_{k}^{n} y_{i} x_{k}\right), \\
\text { s.t } \mathrm{AX} \geq 1 \\
\max \left(f=\sum_{k}^{n} f_{k}\right)
\end{array}\right.
$$

where $x_{k}$ is 1 or 0 , which indicates whether physical grid node $k$ has a PMU installed; $A=\left(a_{i j}\right)$ is the node correlation matrix, where if the two nodes $i, j$ are adjacent or the same, then $a_{i j}=1$ and otherwise, $a_{i j}=0 ; 1$ is the column vector whose elements are all $1 ; f_{k}=x_{k}+\sum_{l \in G} x_{l}$; and $G$ indicates the set of all nodes connected to node $k$. Considering the 39-node grid model, the node correlation matrix is as follows:

$$
A=\left[\begin{array}{c}
A_{1} \\
A_{2} \\
A_{3} \\
\vdots \\
A_{38} \\
A_{39}
\end{array}\right]=\left[\begin{array}{cccccc}
a_{1,1} & a_{1,2} & a_{1,3} & \ldots & a_{1,38} & a_{1,39} \\
a_{2,1} & a_{2,2} & a_{2,3} & \ldots & a_{2,38} & a_{2,39} \\
a_{3,1} & a_{3,2} & a_{3,3} & \ldots & a_{3,38} & a_{3,39} \\
\vdots & \vdots & \vdots & \vdots & \vdots & \vdots \\
a_{38,1} & a_{38,2} & a_{38,3} & \ldots & a_{38,38} & a_{38,39} \\
a_{39,1} & a_{39,2} & a_{39,3} & \ldots & a_{39,38} & a_{39,39}
\end{array}\right]=\left[\begin{array}{cccccc}
1 & 1 & 0 & \ldots & 0 & 1 \\
1 & 1 & 1 & \ldots & 0 & 0 \\
0 & 1 & 1 & \ldots & 0 & 0 \\
\vdots & \vdots & \vdots & \vdots & \vdots & \vdots \\
0 & 0 & 0 & \ldots & 1 & 0 \\
1 & 0 & 0 & \ldots & 0 & 1
\end{array}\right] .
$$
follows:

The cost of installing PMU equipment at node $i$ is as

$$
y_{i}=1+0.1 n_{\mathrm{ch}}
$$

The cost of equipment installation is the sum of a fixed cost and a variable cost, $n_{\mathrm{ch}}$ is the number of PMU channels, indicating the number of observable adjacent lines, generally $1-5$, and the variable cost is increased by 0.1 for each additional line.

According to the above constraint rules, the following formula can be obtained to realize the fully observable IEEE 39 node power network topology: 


$$
\left\{\begin{array}{l}
f_{1}=x_{1}+x_{2}+x_{39} \geq 1, \\
f_{2}=x_{1}+x_{2}+x_{3}+x_{25}+x_{30} \geq 1, \\
f_{3}=x_{2}+x_{3}+x_{4}+x_{18} \geq 1, \\
\vdots \quad \vdots \\
f_{38}=x_{24}+x_{38} \geq 1, \\
f_{39}=x_{1}+x_{9}+x_{39} \geq 1 .
\end{array}\right.
$$

To further explain the impact of the number of channels, the following variables are introduced:

$$
w_{i, j}= \begin{cases}1 & \text { PMU was installed on node } i \text { and made node jobservable, } \\ 0 & \text { Other. }\end{cases}
$$

Then, the above formula can be rewritten to obtain the following inequalities:

$$
\left\{\begin{array}{l}
g_{1}=x_{1}+w_{2,1}+w_{39,1} \geq 1, \\
g_{2}=x_{2}+w_{1,2}+w_{3,2}+w_{25,2}+w_{30,2} \geq 1, \\
g_{3}=x_{3}+w_{2,3}+w_{4,3}+w_{18,3} \geq 1, \\
\quad \vdots \quad \vdots \\
g_{38}=x_{38}+w_{24,38} \geq 1, \\
g_{39}=x_{39}+w_{1,39}+w_{9,39} \geq 1 .
\end{array}\right.
$$

In the case of a single line fault in the physical network, the number of channels on the right side of the fault point at the end of the node line is accordingly changed (i.e., multiplied by 2 to ensure that each node is observed by at least two PMUs). The configuration with the lowest cost is $2,6,8$, $15,16,17,20,23,25,26,29$, and 35 . In the distributed control framework, the sensitivity of the information nodes is considered to select the dominant generator in the area.

2.2. Smart Grid Distributed Framework. According to the information network reliability optimization results and the multiagent system model, a distributed control framework is designed for cooperation between the generator and the external energy storage system in the smart grid. In this framework, the corresponding information and physical equipment at each generator node are combined to form an agent. Each agent represents its main properties by the physical quantities of the generator. The information equipment includes a measurement unit (PMU) and a controller, both of which perform calculations and applications related to information and data. The physical equipment also includes external energy storage equipment in addition to the generator. Only when external energy storage is needed to inject power or absorb a certain power, $P_{L, i}$ will the corresponding controller of the generator agent be activated.

The agents that describe the information-physical network interaction and the system model (Figure 2). The generator participating in stability control of the physical power grid and frequency adjustment without difference is the leading generator, which is realized by the information network through selective control; the power regulation of other generators in the same zone is controlled by the information network to proportionally change with the power of the leading generator to assist in frequency regulation of the leading generator.

The synchronous generators play a leading role in the physical power grid, and thus the stability of the power grid is largely a problem of keeping the interconnected generators synchronized [19]. A smart grid maintains synchronous generator frequency synchronization and phase-angle aggregation by using a distributed control framework based on the multiagent model. According to the requirements of frequency synchronization, the normalized speed corresponding to the grid frequency is selected and denoted by $\omega$, and the generator label in the physical grid is $i \in V, V=$ $\{1,2, \ldots, Z\}$, with

$$
\omega_{i}(t) \longrightarrow 1(t \longrightarrow \infty) \text {. }
$$

To avoid the rapid drop of the node voltage caused by the phase angle difference of the generator terminal voltage exceeding a certain range, thus affecting the safe operation of the unit, the phase angle aggregation requires that generators $i$ and $j$ satisfy the following:

$$
\left|\theta_{i}-\theta_{j}\right| \leq \gamma
$$

where $\gamma$ is a predefined threshold, usually $5 \pi / 9$.

2.3. Smart Grid Control Target Model. The information network selection coefficient $h_{i}$ is set as follows:

$$
h_{i}=\left\{\begin{array}{c}
1, \text { the } i-\text { th agent contains the leading generator, } \\
0, \text { others. }
\end{array}\right\},
$$

This coefficient is used to determine the selective control behaviour of the information network with respect to the physical network and ensure timely control of the main generator agent contained in each partition.

If there is a controller that connects the physical power grid with the information network, then the oscillating equation between motors can be obtained under the constant voltage condition [29]:

$$
\begin{aligned}
M_{i} \dot{\omega}_{i}= & -D_{i} \omega_{i}+P_{m, i}-\left|E_{i}\right|^{2} G_{i i} \\
& -\sum_{j=1, j \neq i}^{Z} P_{i j} \sin \left(\theta_{i}-\theta_{j}+\varphi_{i j}\right)+h_{i} u_{i} .
\end{aligned}
$$

The transient stability problem of a smart grid is described as the control task of multiagent swarm concentration with consistent speed, and then the second-order dynamics model of multiagents is used to describe each agent as follows [30]:

$$
\left\{\begin{array}{l}
\dot{\theta}_{i}=\omega_{i}, \\
D_{i} \dot{\omega}_{i}=-M_{i} \ddot{\omega}_{i}-l_{i j} \omega_{i}+h_{i} \dot{u}_{i} .
\end{array}\right.
$$




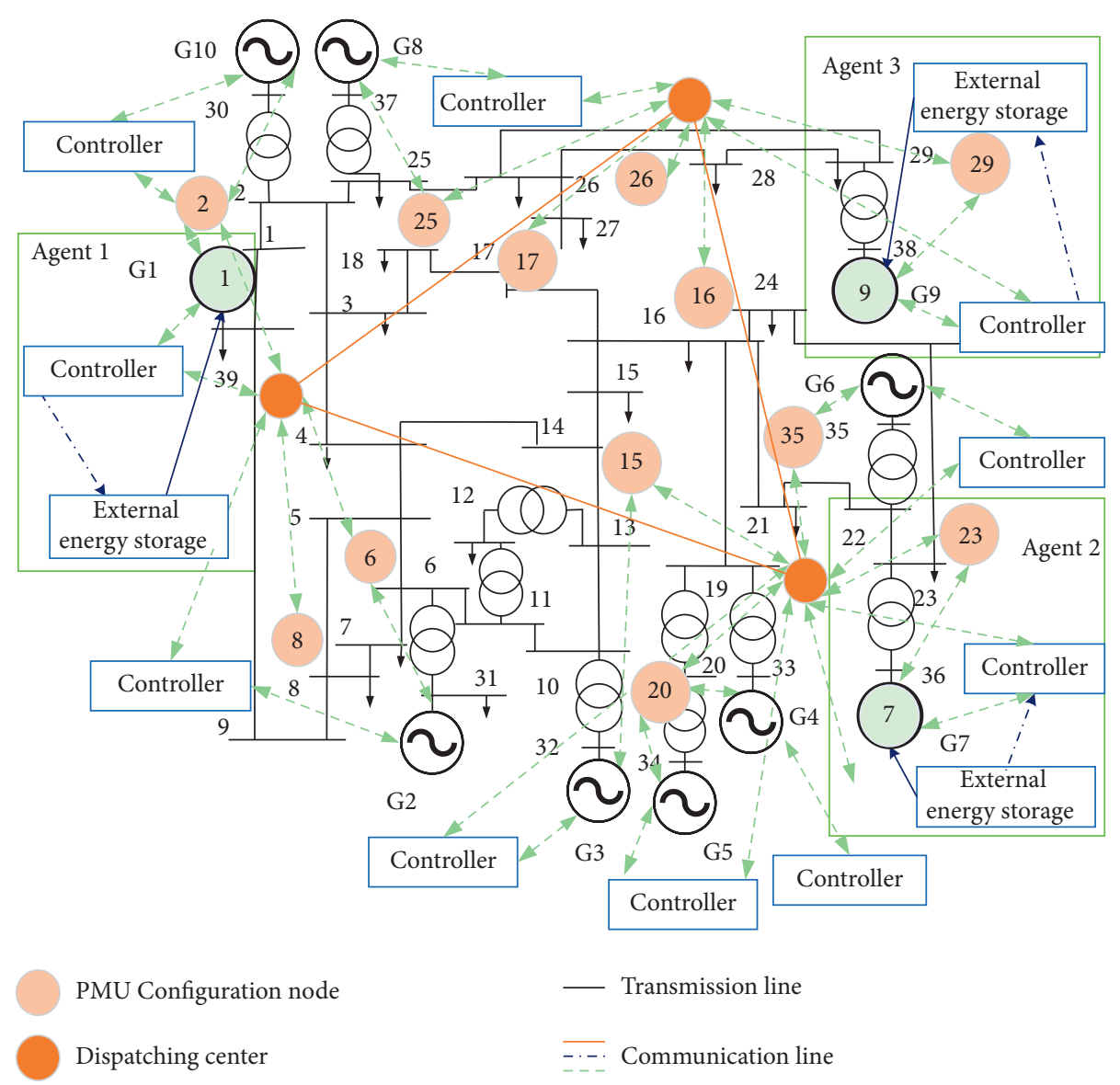

Agent dominant generator node

FIgUre 2: Model of the IEEE 39-bus cyber-physical smart grid with agents.

The controller signal is defined as $u_{i}=P_{L, i}$ for agent $i=1, \ldots, F$, which corresponds to the agent containing the main generator. Since only one main generator is selected for each partition, Falso represents the number of physical power grid zones. $h=\operatorname{diag}\left[h_{1}, \ldots, h_{i}, h_{i+1} \ldots h_{Z}\right]$. Generally, $h_{i}=1$ is set for $i \leq F$; otherwise, $h_{i}=1$ corresponds to other agents in the partition that assist in the adjustment, $V_{\mathrm{mf}}=\{F+i, \ldots, Z\}$. The auxiliary regulated power of the corresponding generator is $P_{F+i}=a_{F+i} \cdot P_{L, i}$, where $a$ is a proportional coefficient that varies with the power of the leading generator and $l_{\mathrm{ij}}$ is an element of the physical relation matrix $L$, which is represented as

$$
l_{i j}=\left\{\begin{array}{l}
\sum_{j=1, j \neq i}^{Z} P_{i j} \cos \left(\theta_{i}-\theta_{j}+\varphi_{i j}\right), i=j . \\
-P_{i j} \cos \left(\theta_{i}-\theta_{j}+\varphi_{i j}\right), i \neq j .
\end{array}\right.
$$

In this case, a new control quantity $\widetilde{u}_{i}$ is introduced to define the controller signal $u_{i}$ as

$$
\dot{u}_{i}=\widetilde{u}_{i}+l_{i j} \omega_{i}-B_{i} \dot{\omega}_{i},
$$

where $B_{\mathrm{i}} \geq\left(100 * D_{i}\right)$ is an element in information relation matrix $B$.

\section{Distributed Area Optimal Control for the Transient Stability of a Smart Grid}

3.1. Smart Grid Area Division. The main properties of each agent in the smart grid are described by the physical quantities of the generators in the physical grid. Let the state information carried by agent $i$ at time $t=k$ be

$$
\left\{\begin{array}{l}
x_{i}(k)=\theta(k), \\
v_{i}(k)=\omega_{i}(k)
\end{array}\right.
$$

When the time step is $t=k, \theta_{i}(k)$ and $\omega_{i}(k)$ are the rotor angle and speed of the $i$-th generator, respectively, which are physical quantities directly obtained from the PMU. $S_{i}(k)=$ $\left[x_{i}(k), v(k)\right]^{T}$ describes the status information carried by individuals, where $\mathrm{x}_{i}(k)$ and $v_{i}(k)$ correspond to the position and speed of the individual at time step $t=k$. If the distance between two individuals is less than the threshold $r$, then they are considered as being in each other's neighbourhood. The neighbourhood set for the $i$-th individual is defined as follows: 


$$
\mathrm{N}_{i}(k)=\left\{j \in V \mid\left\|\mathrm{x}_{i}(k)-x_{j}(k)\right\|<\mathrm{r}\right\} .
$$

According to the similarity of the individual dynamic state quantities, the region is divided; forj $\in \mathrm{N}_{i}(k)$, the relationship between individuals $i$ and $j$ in the same group meets the following requirement:

$$
S_{i}(k)-S_{j}(k)<S_{\mathrm{th}}(k)
$$

where $S_{\text {th }}(k)$ is the set threshold value.

The following dynamic model is used:

$$
x_{i}(k+1)=x_{i}(k)+\Delta t v_{i}(k) .
$$

The physical dynamic quantity of the agent has been transformed into the individual state quantity in the information space, and multiple groups containing different individuals are simultaneously obtained, which are composed of agents with close physical coupling in the actual smart grid corresponding to the partition.

3.2. Smart Grid Distributed Controller. According to formula (13), letting $\underline{M}_{i}=D_{i}+B_{i}$, the second-order system of the set containing the leading generator agent is determined as

$$
\left\{\begin{array}{l}
\dot{\theta}_{i}=\omega_{i} \\
\underline{M}_{i} \dot{\omega}_{i}=\widetilde{u}_{i}
\end{array}\right.
$$

To achieve transient stability of the smart grid, the distributed control signal applied to the agent (label $i=1,2, \cdots, F)$ with the leading generator in each partition is as follows:

$$
\tilde{u}_{i}=-\nabla V\left(\theta_{i j}\right)-\tilde{l}_{i j} \omega_{i}-c\left(\omega_{i}-\omega^{*}\right) .
$$

where $c$ is a constant representing the coefficient of the feedback term; $\omega$ is the expected normalized speed value. $\tilde{l}_{\mathrm{ij}}$ is an element in the velocity matching matrix $\widetilde{L}$, and the first term is the $V\left(\theta_{\mathrm{ij}}\right)$ gradient, where the potential function $V\left(\theta_{\mathrm{ij}}\right)$ is defined as follows:

$$
V\left(\theta_{i j}\right)=\frac{1}{2} \sum_{i=1}^{F} \sum_{j=1, j \neq i}^{F} \chi\left(\theta_{i}-\theta_{j}\right)
$$

The above functions ensure that the rotor angle difference between agents is bounded.

\subsection{Analysis of the Distributed Control Stability of a Smart Grid}

Theorem 1. Consider a second-order system with expected values of the guidance feedback terms in kinetic equations (18) and (19). Suppose that the initial velocity does not match the expected value and that the initial energy $H_{0}$ is finite; under the action of control protocol equation (19), all agents asymptotically and uniformly converge to the corresponding reference speed, corresponding to the expected speed of the generator of $\omega^{*}$, and the group behaviour is consistent with global stability.
Proof. Let $\widetilde{\theta}_{i}=\theta_{i}-\theta^{*}$ and $\widetilde{\omega}_{i}=\omega_{i}-\omega^{*}$ represent the differences between the physical quantities of the agent and the expected values, respectively, then:

$$
\left\{\begin{array}{l}
\dot{\tilde{\theta}}_{i}=\widetilde{\omega}_{i} \\
\underline{M}_{i} \dot{\tilde{\omega}}_{i}=\widetilde{u}_{i}
\end{array}\right.
$$

According to equations (18), (19), and (21), the control function can be obtained as follows:

$$
\tilde{u}_{i}=-\nabla V\left(\tilde{\theta}_{i j}\right)-\tilde{l}_{\mathrm{ij}} \widetilde{\omega}_{i}-c \widetilde{\omega}_{i} .
$$

The Lyapunov function is defined as the total energy of the system, that is, the sum of the total potential energy between agents and the relative potential energy and kinetic energy between the physical quantities of the agents and the expected reference quantities:

$$
H\left(\widetilde{\theta}_{i}, \widetilde{\omega}_{i}\right)=\frac{1}{2} \sum_{i=1}^{F} V\left(\widetilde{\theta}_{i j}\right)+\frac{1}{2} \sum_{i=1}^{F} \widetilde{\omega}_{i}^{T} \underline{M}_{i} \widetilde{\omega}_{i} .
$$

Since the potential function $V\left(\theta_{i j}\right)$ is symmetric with respect to $\theta_{i j}(i, j=1, \ldots, F)$,

$$
\frac{\partial V\left(\widetilde{\theta}_{\mathrm{ij}}\right)}{\partial \widetilde{\theta}_{\mathrm{ij}}}=\frac{\partial V\left(\widetilde{\theta}_{\mathrm{ij}}\right)}{\partial \widetilde{\theta}_{i}}=-\frac{\partial V\left(\widetilde{\theta}_{\mathrm{ij}}\right)}{\partial \widetilde{\theta}_{j}} .
$$

The derivative of equation (23) can be obtained:

$\dot{H}=\frac{1}{2} \sum_{i=1}^{F} \frac{\partial V\left(\widetilde{\theta}_{i j}\right)}{\partial \widetilde{\theta}_{i}}+\sum_{i=1}^{F} \widetilde{\omega}_{i}^{T} \underline{M}_{i} \dot{\tilde{\omega}}_{i}=\sum_{i=1}^{F} \widetilde{\omega}_{i}^{T} \nabla V\left(\widetilde{\theta}_{i j}\right)+\sum_{i=1}^{F} \widetilde{\omega}_{i}^{T} \widetilde{u}_{i}$.

Equation (22) can be substituted into equation (25) to obtain

$$
\begin{aligned}
\dot{H} & =\sum_{l=1}^{F} \widetilde{\omega}_{i}^{T} \nabla V\left(\widetilde{\theta}_{i j}\right)-\sum_{i=1}^{F} \widetilde{\omega}_{i}^{T} \nabla V\left(\widetilde{\theta}_{i j}\right)-\sum_{i=1}^{F} \widetilde{\omega}_{i}^{T} \widetilde{l}_{i j} \widetilde{\omega}_{i}-\sum_{i=1}^{F} \widetilde{\omega}_{i}^{T} c \widetilde{\omega}_{i} \\
& =-\sum_{i=1}^{F} \widetilde{\omega}_{i}^{T} \widetilde{l}_{i j} \widetilde{\omega}_{i}-\sum_{i=1}^{F} \widetilde{\omega}_{i}^{T} c \widetilde{\omega}_{i}=-\sum_{i=1}^{F} \widetilde{\omega}_{i}^{T}\left[\left(\widetilde{l}_{i j}+c\right)\right] \widetilde{\omega}_{i} \\
& =-\widetilde{\omega}^{T}[(\widetilde{L}+c I)] \widetilde{\omega} .
\end{aligned}
$$

Since $\widetilde{L}$ is a semipositive definite Laplace matrix and $c>0, \widetilde{L}+c I$ is a positive definite matrix, and thus, $\widetilde{\omega}^{T}[(\widetilde{L}+$ $c I)] \widetilde{\omega}>0$ and $\dot{H}<0$. Therefore, the agents asymptotically stably converge to their expected reference values under control input (19).

\section{Simulation Example}

In this paper, the IEEE 39 node smart grid model is analysed as an example, which includes 19 loads and 46 lines, which are simulated by the MATLAB/Simulink platform. According to the classification of a short-circuit fault, centralized control after a fault and swarm control after a fault, the examples are divided into four scenarios. 


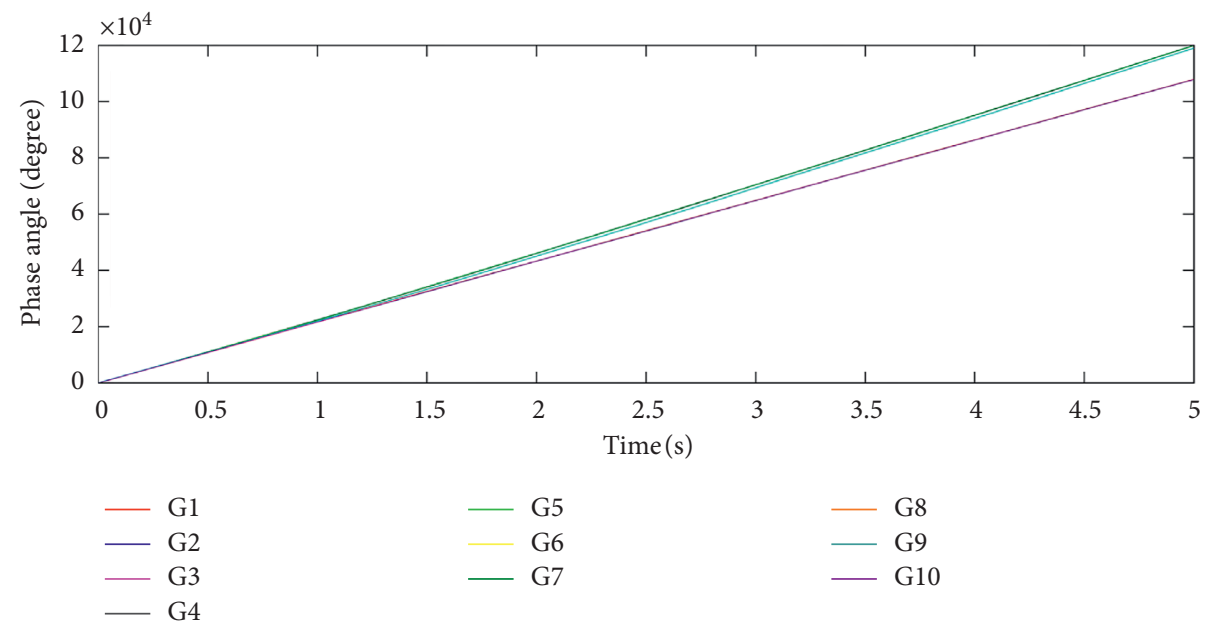

(a)

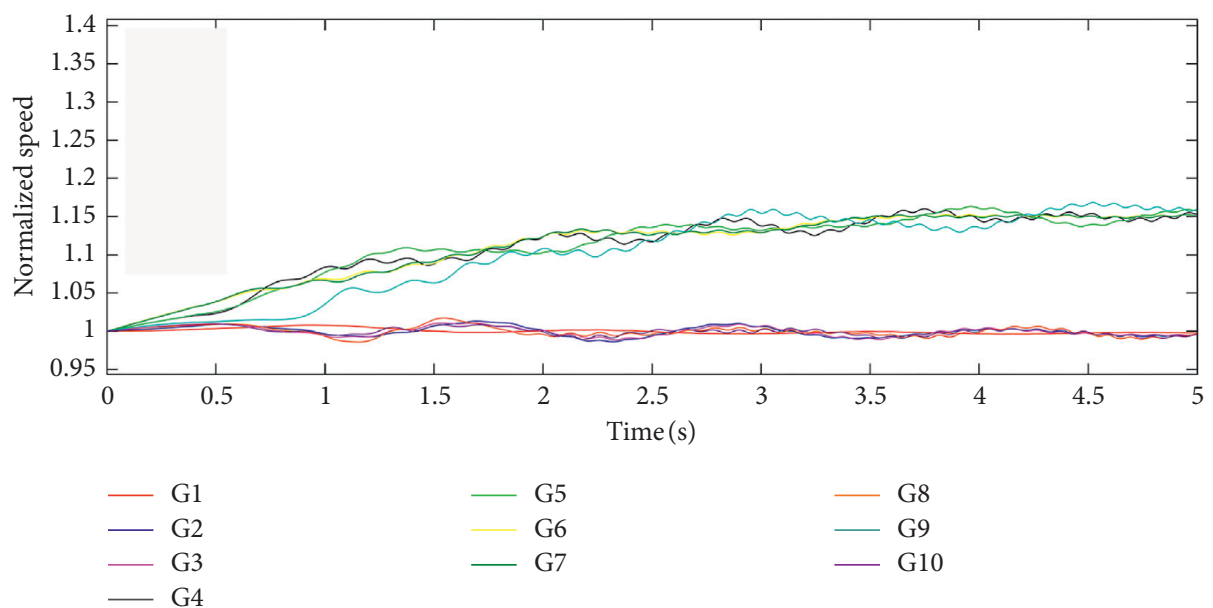

(b)

Figure 3: Relative rotor angles and rotor speeds of generators after the fault.

Scenario 1. Suppose at $t=0 \mathrm{~s}$, a three-phase short-circuit fault occurs on disturbed line 21-22 of the power grid. Disconnect the fault line and set $h_{i}=0$ for $i \in V, V=\{1,2, \cdots, Z\}$, that is, the controller connecting the information and physical networks is not activated and the information network traffic and calculation amount are minimal. The relationships between the speeds of each generator and the rotor angles are given in Figure 3. The system operation is obviously unstable, the rotor angle has an increasing difference trend, and the power network has a serious fault.

Scenario 2. Under centralized control, each node of the power grid needs to transmit information to the dispatching centre. After centralized processing, a large number of PMUs and communication lines are needed to ensure information collection and transmission. In the period before centralized control after fault removal, it is necessary to collect the state information of the whole power grid, assuming that the data can be collected and processed within the preset critical time after fault treatment under ideal conditions. When $t=0.15 s$, set $h_{i}=0$ for $i \in V$,
$V=\{1,2, \cdots, Z\}$, so that all generator nodes are controlled by no difference regulation. Under ideal conditions(Figure 4), the rotor angles and speeds of the generators change with time and the system achieves a stable state in a short time. However, the system is in the state of maximum information transmission and processing. The control equipment of each generator node frequently uses external energy devices to adjust the power of the corresponding nodes. The overall energy consumption of the whole regulation process is large, and the network topology and performance of the related equipment are not fully utilized.

Scenario 3. The flocking control method leads to a stable performance (Figure 5). Distributed flocking control can reduce the equipment cost and improve the centralized control of information processing, the energy consumption, and the critical stability range in the $5 \mathrm{~s}$ of the observation period, but the fluctuation range is larger. The smart grid after using each agent to facilitate control tends to be stable for a long time. 


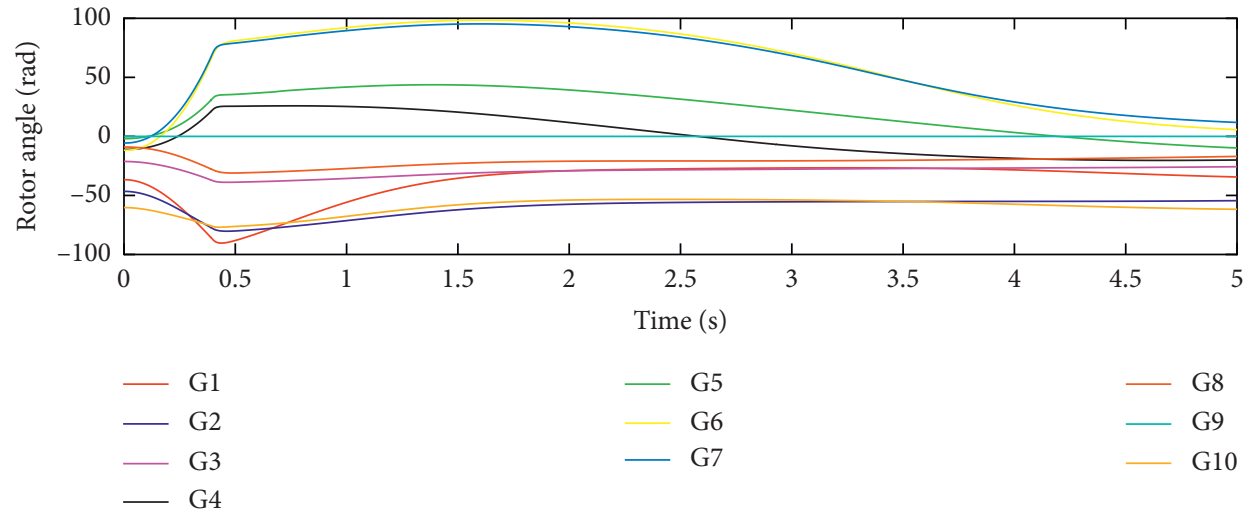

(a)

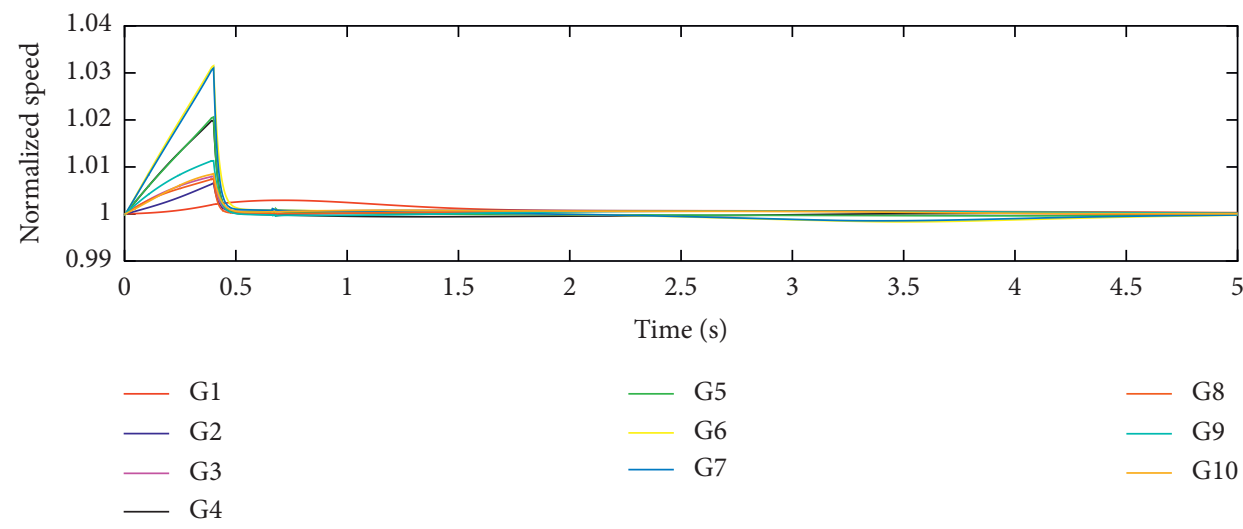

(b)

FIGURE 4: Relative rotor angles and rotor speeds of generators under centralized control after the fault.

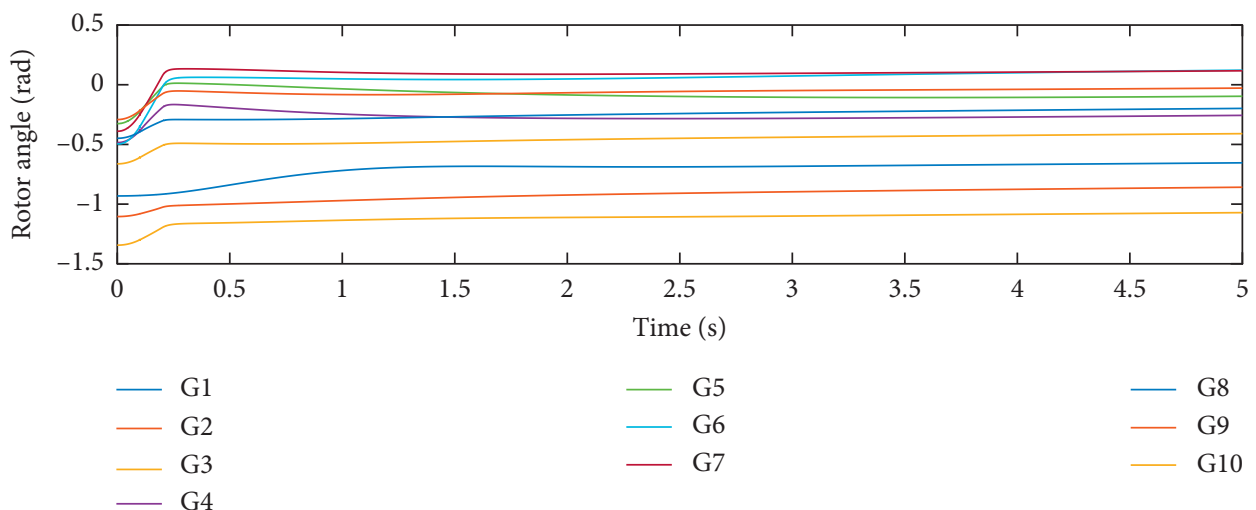

(a)

Figure 5: Continued. 


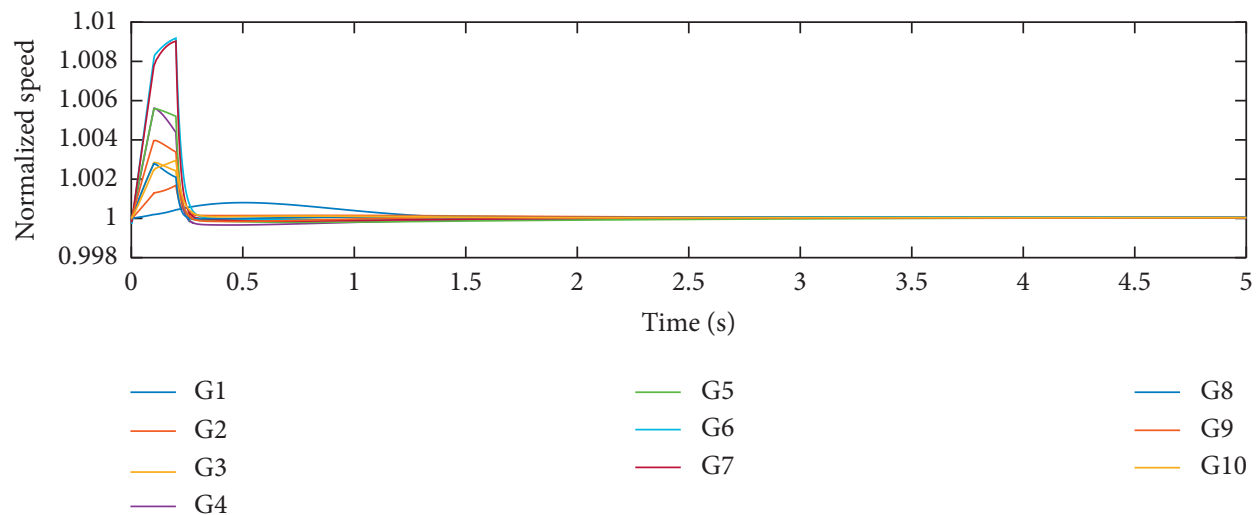

(b)

FIGURE 5: Relative rotor angles and rotor speeds of generators under flocking control after the fault.

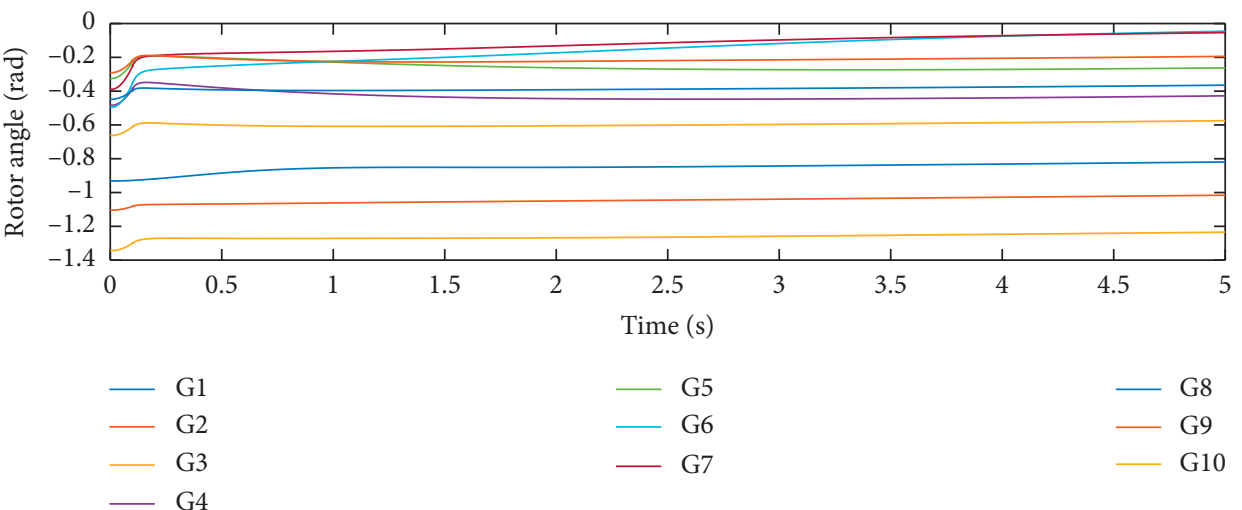

(a)

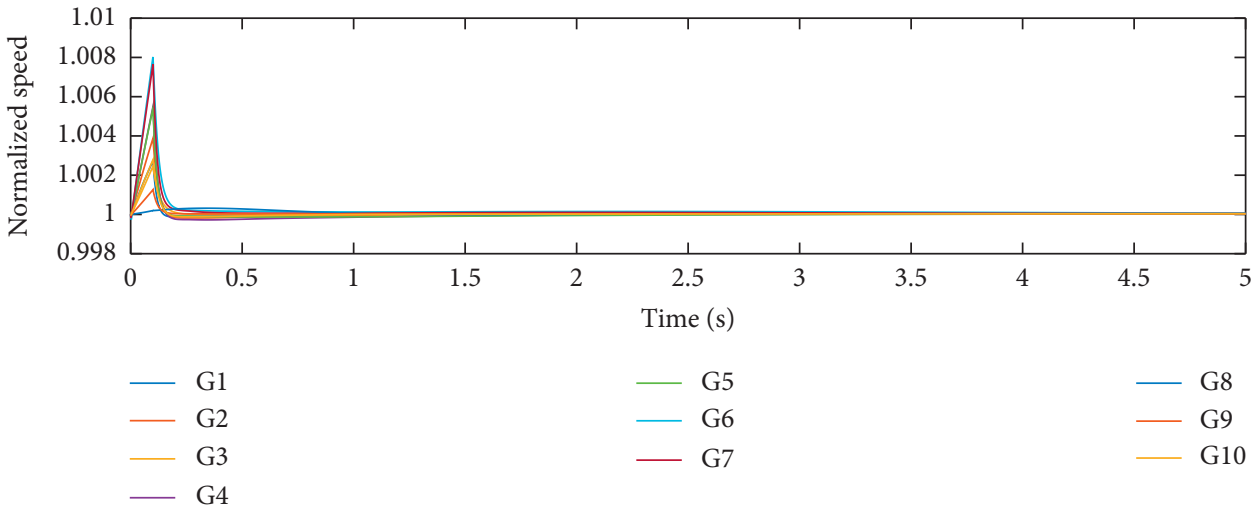

(b)

FIGURE 6: Relative rotor angles and rotor speeds of generators under distributed optimal control after the fault.

Scenario 4. The distributed optimal control method in this paper is used to control the performance (Figure 6). The smart grid partition identification scheme should be enabled within the preset critical time point after fault treatment to avoid more damage and improve the ability of the smart grid to restore transient stability. The distributed optimal control is applied to the agents containing the main generators, that is, setting $h_{i}=1$ for $i=1, \ldots, F$.
First, determine the smart grid partition based on the similarity of the intelligent agent dynamic characteristics; that is, the smart grid agent quantity changes corresponding to the movement trend of individuals in the information space. Next, obtain the state information of individual trajectories, described as individual motion curves in a twodimensional space. From the trend of separation of individuals in the information space, that is, the change of the 
physical quantities of each agent after the failure, it can be found which individuals are obviously far from other individuals and which movement trends are the closest. The following partitions can be obtained from the partitioning scheme of dynamic recognition of each agent: $\{G 1\}$, $\{G 2 G 3 G 8 G 9 G 10\}$, and $\{G 4 G 5 G 6 G 7\}$.

Second, on the basis of information network reliability optimization combined with the actual active power output for each agent and sensitivity weight arrangement of the node data, reasonable leading generators $G 1, G 7$, and $G 9$ are selected for each partition, and distributed optimal control is applied to the intelligent agent with the leading generator to achieve the goal of system transient stability.

Within the critical time after the fault is cut off, partition identification and leading generator selection are completed. At $t=0.15 \mathrm{~s}$, the distributed control scheme combining the information and physical networks is applied to the smart grid. The state quantities of each agent change with time (Figure 6). The control objective is achieved for each agent in each partition, and the corresponding physical quantity converges to a certain value according to the partition. Since external energy injection could impact actual operation and lead to certain fluctuations, we quickly recover to the stable operation of the steady-state power system. The generator speeds are stable and gradually converge, and the rotor angles satisfy the requirements of the constraint and stable state. Under the conditions of information use and smaller external energy injection, the good effect of a shorter stable system recovery time, a smaller physical quantity fluctuation range, and an increased stability margin of the system are achieved.

Under the action of centralized control method, the system can resume stable operation in a short time, but the disadvantage is that the system is in the state of maximum information transmission and processing and the overall energy consumption is large. Compared with centralized control, distributed swarm control improves the disadvantages of large amount of information processing and energy consumption, but the time of system stability is prolonged. The distributed optimal control method proposed in this paper can quickly restore the stability of the system, reduce the energy consumption, and improve the stability margin of the smart grid.

\section{Conclusions}

In this paper on transient stability control of the smart grid model, the distributed multiagent control method adopted in the power grid model after reliability optimization of the information network is described. In the distributed control framework, the dynamic similarity of the physical parameters of the generators is used to form each zone, and external energy storage is used to adjust the agents in each zone. The leading generator realizes no difference adjustment, and other generators in the same zone adjust their power proportional to the adjusted power of the leading generator to achieve asymptotic stability of each zone. The simulation results in this paper verify the effectiveness of the proposed control scheme, which finally achieves transient stability and control of the smart grid model, and it has fast and stable performance compared with swarm control. The idea presented in this paper has a certain reference value for improving the transient stability of smart grids in practical projects.

\section{Data Availability}

The data used to support the findings of this study are included within the article.

\section{Conflicts of Interest}

The authors declare that they have no conflicts of interest.

\section{Acknowledgments}

This work was supported in part by the National Natural Science Foundation of China under Grant no. 11662002.

\section{References}

[1] S. Y. Chen, S. F. Song, and L. X. Li, "Survey on smart grid technology," Power System Technology, vol. 33, no. 8, pp. 1-7, 2009.

[2] P. Kundur, N. J. Balu, and M. G. Lauby, Power System Stability and Control, McGraw-Hill, New York, NY, USA, 1994.

[3] H. Li, Z. Han, A. D. Dimitrovski, and Z. Zhang, "Data traffic scheduling for cyber physical systems with application in voltage control of distributed generations: a hybrid system framework," IEEE Systems Journal, vol. 8, no. 2, pp. 542-552, 2014.

[4] E. Mengelkamp, B. Notheisen, C. Beer et al., "A blockchainbased smart grid: towards sustainable local energy markets," vol. 33, no. 1-2, pp. 207-214, Computer Science Research \& Development, 2018.

[5] K. Muralitharan, R. Sakthivel, and R. Vishnuvarthan, "Neural network based optimization approach for energy demand prediction in smart grid," Neurocomputing, vol. 273, pp. 199-208, 2018.

[6] Q. Y. Sun, F. Teng, H. G. Zhang et al., "Construction of dynamic coordinated optimization control system for energy internet," Proceedings of the CSEE, vol. 14, pp. 3667-3677, 2015.

[7] D. Carrion and J. W. Gonzalez, "Optimal PMU location in electrical power systems under N-1 contingency," in Proceedings of the International Conference on Information Systems and Computer Science, INCISCOS) IEEE Computer Society, Kuala Lumpur, Malaysia, March 2018.

[8] E. Abiri, F. Rashidi, and T. Niknam, "An optimal PMU placement method for power system observability under various contingencies," International Transactions on Electrical Energy Systems, vol. 25, no. 4, pp. 589-606, 2015.

[9] F. Rashidi, E. Abiri, T. Niknam, and M. R. Salehi, "Optimal placement of PMUs with limited number of channels for complete topological observability of power systems under various contingencies," International Journal of Electrical Power \& Energy Systems, vol. 67, pp. 125-137, 2015.

[10] Z. Wang and W. Jie, "A novel finite-time control scheme for enhancing smart grid frequency stability and resilience," IEEE Transactions on Power Systems, vol. 10, no. 6, pp. 6538-6551, 2019.

[11] A. Q. Huang, M. L. Crow, G. T. Heydt, J. P. Zheng, and S. J. Dale, "The future renewable electric energy delivery and management (FREEDM) system: the energy internet : the 
energy internet," Proceedings of the IEEE, vol. 99, no. 1, pp. 133-148, 2011.

[12] Z. Li, W. Ren, X. Liu, and M. Fu, "Distributed containment control of multi-agent systems with general linear dynamics in the presence of multiple leaders," International Journal of Robust and Nonlinear Control, vol. 23, no. 5, pp. 534-547, 2013.

[13] D. U. Jia-Bing, C. Yan-Peng, L. Man-Fa et al., "Design and implementation of smart grid control system based on distributed real-time scheduling strategy," Electronic Design Engineering, vol. 26, no. 7, pp. 119-127, 2018.

[14] Y. Xu, W. Zhang, M.-Y. Chow, H. Sun, H. B. Gooi, and J. Peng, "A distributed model-free controller for enhancing power system transient frequency stability," IEEE Transactions on Industrial Informatics, vol. 15, no. 3, pp. 1361-1371, 2019.

[15] Y. Zhou, K. Wang, and G. Li, "Distributed hierarchical control for microgrid based on multi-agent consensus Algorithm," Automation of Electric Power Systems, vol. 41, no. 11, pp. 142-149, 2017.

[16] G. Dekker and J. Frunt, Frequency Stability Contribution of Wartsila Combustion on Engines, Wartsila Finland OY, Vaasa, Finland, 2012.

[17] A. Farraj, E. Hammad, D. Kundur et al., "On the use of energy storage systems and linear feedback optimal control for transient stability," IEEE Transactions on Industrial Informatics, vol. 13, no. 4, pp. 1575-1585, 2017.

[18] H. Silva-Saravia, H. Pulgar-Painemal, and J. Mauricio, "Flywheel energy storage model, control and location for improving stability: the Chilean case," IEEE Transactions on Power Systems, vol. 1, no. 1, p. 99, 2016.

[19] K. Kawabe and A. Yokoyama, "Improvement of transient stability and short-term voltage stability by rapid control of batteries on EHV network in power systems," Electrical Engineering in Japan, vol. 188, no. 3, pp. 1-10, 2014.

[20] S. Baros and M. D. Ili, "A consensus approach to real-time distributed control of energy storage systems in wind farms," IEEE Transactions on Smart Grid, vol. 10, no. 1, pp. 613-625, 2019.

[21] W. Ziqiang and W. Jie, "A distributed control considering the cyber-physical characteristics of smart grid," Proceedings of the CSEE, vol. 39, no. 23, pp. 6921-6933, 2019.

[22] S. Chen, H. Pei, Q. Lai et al., "Multitarget tracking control for coupled heterogeneous inertial agents systems based on flocking behavior," in Proceedings of the IEEE Transactions on Systems Man \& Cybernetics Systems, pp. 1-7, January 2018.

[23] C. Shiming, G. Junjie, G. Yanli et al., "Observer-based eventtriggered tracking consensus of non-ideal general linear multi-agent systems," Journal of the Franklin Institute, vol. 356, no. 17, 2019.

[24] H. Pei, S. Chen, and Q. Lai, "Multi-target consensus circle pursuit for multi-agent systems via a distributed multiflocking method," International Journal of Systems Science, vol. 47 , no. 16, 2016.

[25] H. Pei, S. Chen, Q. Lai et al., "Consensus tracking for heterogeneous interdependent group systems," IEEE Transactions on Cybernetics, vol. 50, no. 4, 2020.

[26] Z. Zhang, S. Chen, and H. Su, "Scaled consensus of secondorder nonlinear multiagent systems with time-varying delays via aperiodically intermittent control," IEEE Transactions on Cybernetics, no. 99, pp. 1-14, 2019.

[27] A. MOEINI, I. KAMWA, P. BRUNELLE et al., Open Data IEEE Test Systems Implemented in SimPowerSystems for Education and Research in Power Grid Dynamics and Control, in
Proceedings of the 2015 50th International Universities Power Engineering Conference, pp. 1-6, Stroke-on-Trent, UK, September 2015.

[28] Z. Zhuoli, Y. Ping, X. U. Zhirong et al., "Review on largedisturbance transient stability research of microgrid with multiple energy resources and multiple conversions," Power System Technology, vol. 41, no. 7, pp. 2195-2204, 2017.

[29] F. Dorfler, M. Chertkov, and F. Bullo, "Synchronization in complex oscillator networks and smart grids," Proceedings of the National Academy of Sciences, vol. 110, no. 6, pp. 20052010, 2013.

[30] R. Olfati-Saber, "Flocking for multi-agent dynamic systems: algorithms and theory," IEEE Transactions on Automatic Control, vol. 51, no. 3, pp. 401-420, 2006. 